\title{
Zur Goethe-Literatur
}

J.W.Goethe, Naturwissenschaftliche Schriften. I. Teil: Schriften zur Farbenlehre. Gedenkausgabe der Werke, Briefe und Gespräche, herausgegeben von E. Beutler. Band 16. Zürich, Artemis-Verlag, 1949.

Dem stattlichen Band ist eine Einführung von ANDreas Speiser beigegeben, welche die ideengeschichtliche und physikalische Seite der «Farbenlehre» in ihrer typischen Problemstellung des 18. Jahrhunderts und als bedeutendste wissenschaftliche Leistung GoEthes aus tiefer eigener Kenntnis zur Darstellung bringt.

Ist es nicht leicht, GoEtHEs Farbenlehre in ihrer geistesgeschichtlichen Bedeutung dem heutigen Gebildeten, noch weniger dem Physiker von Fach, nahezubringen, so ist es um so verdienstlicher, wenn ein so eng mit der Materie Vertrauter wie ANDreas Speiser sich die Mühe nimmt, auf dem Wege einer wahren Introduktion in das Wesen und in das Wesentliche von GoEthes Farbenlehre dem Leser die Möglichkeit eines vertiefteren Verständnisses zu vermitteln.

Bleibt die Physiologische Optik GoEtHEs für alle Zeiten ein wissenschaftliches Denkmal mit der Sinnesphysiologie und ihren wissenschaftlichen Anfängen untrennbar verbunden, so erregt auch in den Teilen, welche von der exakten Physik abgelehnt werden, die fabelhafte experimentelle Leistung Góethes und die phänomenologische Beschreibung seiner Beobachtungen auch heute unsere Bewunderung.

Jedem Freund der naturwissenschaftlichen Schriften GoEthes und der Geschichte der Naturwissenschaften überhaupt sei diese besonders wertvolle Ausgabe der Farbenlehre ans Herz gelegt. Ein zweiter abschließender Teil der naturwissenschaftlichen Schriften GoEtHEs befindet sich in dieser schönen Ausgabe bereits in Vorbereitung.

H. Fischer

Goethe, Die Schriften zur Naturwissenschaft. Vollständige, mit Erläuterungen versehene Ausgabe herausgegeben im Auftrage der Deutschen Akademie der Naturforscher zu Halle von Günther Schmid, Wilhelm Troll und Lothar Wolf. Erste Abteilung: Texte. Erster Band: Schriften zur Geologie und Mineralogie 1770-1810. 391 Seiten mit 20 Tafeln. Weimar 1947. Zweiter Band: Schriften zur Geologie und Mineralogie 1812-1832. 438 Seiten mit 34 Tafeln. Weimar, Hermann Böhlaus Nachfolger, 1949. Beide Bände herausgegeben von GüNTHER SCHMID.

Die Bände enthalten den Text in chronologischer Folge und in einer die Sophienausgabe übertreffenden Vollständigkeit. Band 1 ist als Quellenmaterial für die Geschichte des Ilmenauer Bergbaues und den Anteil GoEthes daran von größtem 
Wert. Eine Beurteilung der mit Bildtafeln vorzüglich ausgestatteten Ausgabe wird erst möglich sein, wenn die zugehörigen Erläuterungen vorliegen.

\section{H. Fischer}

Goethe, Schriften über die Natur. Geordnet und ausgewählt von Gunther Ipsen, Stuttgart, Alfred Kröner Verlag, 1949 (Kröners Taschenausgabe Band 62). 343 Seiten. Kurze Auswahl der wichtigsten Texte mit einer knappen Einleitung.

H. Fischer

Bernhard Peyer, Goethes Wirbeltheorie des Schädels. Neujahrsblatt, herausgegeben von der Naturforschenden Gesellschaft in Zürich auf das Jahr 1950. Zürich, Kommissionsverlag Gebr. Fretz, 1950. 129 Seiten mit 87 Abbildungen.

Zur Behandlung dieses schon wiederholt erörterten Problems könnte man sich keinen geeigneteren Bearbeiter denken als B. PEYER, dessen vergleichend-anatomische und historische Zuständigkeit den Autor geradezu prädestiniert, in dieses umstrittene Thema neue Klarheit zu bringen, und bei aller Verehrung Goethes die durch die Wissenschaft gebotene kritische Grenzsetzung am richtigen Ort zu ziehen. Dies betrifft beispielsweise auch die von manchen Morphologen viel zu extensiv deszendenztheoretisch ausgelegte Auffassung einiger vager Aussprüche Goethes, welche gerade zeigen, daß Gozthe dieser Gesichtspunkt fast völlig fernlag.

In einem einführenden Kapitel gibt PEyer einen gedrängten Überblick über GoEthes morphologische Schädelstudien und seine Entdeckung des Zwischenkiefers beim Menschen, welche GoEthe in helle Begeisterung versetzte, von der anatomischen Fachwelt aber mit Stillschweigen bedacht wurde oder kränkende Ablehnung erfuhr, was GoEthe veranlaßte, seine sorgfältig redigierte und illustrierte Abhandlung erst etwa ein halbes Jahrhundert (1831) nach ihrer Entstehung zu publizieren. Peyer stellt das Problem der Entdeckung des Zwischenkiefers, dessen Existenz schon in viel früherer Zeit bald bestritten, bald angenommen wurde, dadurch auf eine breitere Grundlage, daß er (Kapitel II) auch die schädelmorphologischen Arbeiten von Zeitgenossen GoEтHEs, insbesondere die bekannten Arbeiten VIc. D'Azyrs, beizieht. Das eigentliche Thema wird mit OKens Wirbeltheorie des Schädels eingeleitet und an den phantastischen Wirbelkonstruktionen eines C. G. CARUs eigentlich ad absurdum geführt. Ein III. Kapitel verfolgt die Probleme der Schädelmorphologie im historischen Rückblick, und daran anschließend Kapitel IV, «Die Zeit von Goethes Tod bis zu TH. H.HuxLEys Croonian Lecture (1858)» umfassend, entwickelt PEYer auf eingehender historischer Literaturkenntnis basierend und mit dem Rüstzeug des modernen vergleichenden Anatomen ausgestattet, die weiteren Auswirkungen der osteo- 